\title{
Two new endophytic species enrich the Coniochaeta endophytica / C. prunicola clade: Coniochaeta lutea sp. nov. and C. palaoa sp. nov.
}

\author{
A. Elizabeth Arnold ${ }^{1,2^{*}}$, Alison H. Harrington ${ }^{2}$, Jana M. U'Ren ${ }^{3}$, Shuzo Oita ${ }^{1}$ \\ \& Patrik Inderbitzin ${ }^{4}$
}

\author{
Article info \\ Received: 18 Nov. 2021 \\ Revision received: 7 May 2021 \\ Accepted: 7 Jul. 2021 \\ Published: 30 Jul. 2021
}

Associate Editor

Marcin Piątek

\begin{abstract}
Coniochaeta (Coniochaetaceae, Ascomycota) is a diverse genus that includes a striking richness of undescribed species with endophytic lifestyles, especially in temperate and boreal plants and lichens. These endophytes frequently represent undescribed species that can clarify evolutionary relationships and trait evolution within clades of previously classified fungi. Here we extend the geographic, taxonomic, and host sampling presented in a previous analysis of the clade containing Coniochaeta endophytica, a recently described species occurring as an endophyte from North America; and C. prunicola, associated with necroses of stonefruit trees in South Africa. Our multi-locus analysis and examination of metadata for endophyte strains housed in the Robert L. Gilbertson Mycological Herbarium at the University of Arizona (ARIZ) (1) expands the geographic range of C. endophytica across a wider range of the USA than recognized previously; (2) shows that the ex-type of C. prunicola (CBS 120875) forms a well-supported clade with endophytes of native hosts in North Carolina and Michigan, USA; (3) reveals that the ex-paratype for C. prunicola (CBS 121445) forms a distinct clade with endophytes from North Carolina and Russia, is distinct morphologically from the other taxa considered here, and is described herein as Coniochaeta lutea; and (4) describes a new species, Coniochaeta palaoa, here identified as an endophyte of multiple plant lineages in the highlands and piedmont of North Carolina. Separation of CBS 120875 and CBS 121445 into C. prunicola sensu stricto and C. lutea is consistent with previously described genomic differences between these isolates, and morphological and functional differences among the four species (C. endophytica, C. prunicola, C. palaoa, and C. lutea) underscore the phylogenetic relationships described here. The resolving power of particular loci and the emerging perspective on the host- and geographic range of Coniochaeta and the C. endophytica / C. prunicola clade are discussed.
\end{abstract}

Key words: actin, anamorph, barcode, biodiversity, endolichenic, Lecythophora

\section{Introduction}

Fungal endophytes occur in healthy tissues of plants worldwide (Rodriguez et al. 2009). In contrast to fungi that readily produce visible structures on host material or substrates, the diverse endophytes that occur within symptomless tissues of plants and lichens have only recently begun to receive more active attention from systematists (see Bussaban et al. 2003; Rojas et al. 2008, 2010; Gazis et al. 2011, 2012; Bills et al. 2013; Chen

\footnotetext{
${ }^{1}$ School of Plant Sciences, The University of Arizona, Tucson, AZ 85721 USA

2 Department of Ecology and Evolutionary Biology, The University of Arizona, Tucson, AZ 85721 USA

${ }^{3}$ Biosystems Engineering, The University of Arizona, Tucson, AZ 85721 USA

${ }^{4}$ Indigo Agriculture, Boston, MA 02129 USA

* Corresponding author e-mail: arnold@ag.arizona.edu
}

et al. 2015; Torres-Cruz et al. 2017; Harrington et al. 2019). A relative lack of studies prior to the past three decades means that endophytes are under-represented in culture collections and may be overlooked in evolutionary or taxonomic treatments, even if they are represented in GenBank with barcode sequences. Moreover, the prevalence of saprotrophic and pathogenic fungi in collections and databases often leads to casual assignment of their species names to newly discovered endophytes, even though careful exploration can demonstrate the uniqueness and often novelty of the endophytic taxa (Harrington et al. 2019). Including endophytes in phylogenetic and taxonomic studies provides insight with regard to the identification of endophytes while informing the diversity, relationships, and ecological traits ascribed to previously described taxa (see U'Ren et al. 2016; 
Carbone et al. 2016; Torres-Cruz et al. 2017; Harrington et al. 2019).

Temperate and boreal plants and lichens frequently harbor endophytes in the genus Coniochaeta (Coniochaetaceae; Coniochaetales; Sordariomycetes, Ascomycota). Diverse strains can be isolated readily from healthy, surface-sterilized tissues on standard media such as malt extract agar (MEA) (e.g., Arnold \& Hoffman 2008; U'Ren et al. 2012; del Olmo Ruiz 2012; Huang et al. 2016; Chen 2017; Harrington et al. 2019; U'Ren et al. 2019; Oita et al. 2021). As described in Harrington et al. (2019), Coniochaeta is a diverse genus with species that inhabit a wide range of substrates, including butter, dung, wood, soil, uranium mine wastewater, lichens, and healthy, diseased, or senescent tissues of mosses, ferns, conifers, and angiosperms (see also Weber 2002; García et al. 2006; del Olmo Ruiz 2012; Rafa et al. 2012; Vázquez-Campos et al. 2014; Xie et al. 2015; Chen 2017; U'Ren et al. 2019). Harrington et al. (2019) also notes the teleomorph-anamorph connection of Coniochaeta and Lecythophora, significant in this context because of the high isolation frequency of morphologically cryptic, asexually reproducing fungi in endophyte surveys (Melin \& Nannfeldt 1934; Gams \& McGinnis 1983; Weber 2002; del Olmo Ruiz 2012; Khan et al. 2013; Réblová et al. 2016).

As presently known, Coniochaeta includes between $\sim 50$ and 120 species, depending on the taxonomic resource consulted (see García et al. 2006; Asgari et al. 2007; Kirk et al. 2008, and online sources such as Species Fungorum and MycoBank; see also discussion in Harrington et al. 2019). As a result, it can be challenging to determine whether newly collected endophytes that fall within Coniochaeta represent novel species or are members of known taxa, especially if strains remain sterile in culture, if only the barcode locus is sequenced, and/or if sequences and specimens of named species are not readily available in public databases or biodiversity collections. As noted by Friebes et al. (2016), many publicly available sequences labeled as Coniochaeta are not robustly identified or are misidentified, and voucher specimens often are lacking. Moreover, the barcode locus for fungi (nuclear ribosomal internal transcribed spacers and 5.8S, ITS rDNA) can be highly similar or identical between closely related species of Coniochaeta, necessitating multilocus or phylogenomic analyses to distinguish closely related taxa (see Nasr et al. 2018; Harrington et al. 2019).

Harrington et al. (2019) explored these issues in a morphological, multilocus, and phylogenomic analysis of endophytes with a close phylogenetic affiliation to Coniochaeta prunicola. In that study, two strains isolated as endophytes from healthy, mature foliage of Platycladus orientalis (Cupressaceae) in an arboretum in Arizona, USA were found to be affiliated with, but distinct from, the ex-type and paratype of C. prunicola (CBS 120875 and CBS 121445, respectively; Damm et al. 2010). The strains representing $C$. prunicola occurred in association with necrotic wood of stonefruit trees in South Africa (Prunus armeniaca and P. persica, respectively) (see Damm et al. 2010; see also Ivanová \& Bernadovičová 2012). Harrington et al. (2019) concluded that the endophytic strains of interest from Arizona represented a novel species therein described as C. endophytica. That study relied on two loci, with corroborating evidence from genomic data, in vitro assays, and morphological characterization.

As an extension of that study we sought to classify additional isolates that were obtained as endophytes from diverse plants and lichens (Table 1) and accessioned as part of the living collection of endophytes at the Robert L. Gilbertson Mycological Herbarium of the University of Arizona (ARIZ) (Hoffman \& Arnold 2008; U'Ren et al. 2012; U'Ren et al. 2019; Oita et al. 2021). Sequencing of the barcode locus for these isolates suggested that they are members of the C. prunicola / C. endophytica clade, but their relationships and species identities could not be inferred from ITS rDNA data alone.

The goals of this study were to determine the taxonomic placement of 17 endophytes isolated from plants and lichens of North America and Eurasia, and to evaluate how data from additional loci might clarify relationships within the C. prunicola / C. endophytica lineage in cases in which genome-scale data are not available. Our work provides evidence based on multiple loci, coupled with morphological data, that the C. prunicola / C. endophytica clade contains at least four species: $C$. endophytica, for which this study expands the number of known isolates and the known geographic range; $C$. prunicola, here enriched with endophytes and retaining the ex-type CBS 120875; and two new species. The resolving power of particular loci and emerging perspectives on the host- and geographic range of the clade are discussed.

\section{Materials and methods}

Endophytes were isolated from living, apparently healthy photosynthetic tissues of plants and lichens (Table 1) as described by Hoffman and Arnold (2008), U'Ren et al. (2012), U'Ren et al. (2019), and Oita et al. (2021). Briefly, fresh tissues were rinsed in running tap water for $30 \mathrm{~s}$ and then cut into $2 \mathrm{~mm}$ pieces. Tissue pieces were agitated in $95 \%$ ethanol for $30 \mathrm{~s}, 10 \%$ bleach $(0.5 \% \mathrm{NaOCl})$ for $2 \mathrm{~min}$, and $70 \%$ ethanol for $2 \mathrm{~min}$. After surface-drying under sterile conditions, pieces were placed on $2 \%$ MEA and incubated at $25^{\circ} \mathrm{C}$ for up to 1 year. All isolates that emerged were transferred to axenic culture and vouchered at ARIZ (isolation codes indicate accession numbers; key sequences also are available at MycoBank). ITS rDNA was sequenced for each isolate as described previously (Hoffman \& Arnold 2008; U'Ren et al. 2012). Manually edited, bidirectional sequences were identified tentatively on the basis of BLAST matches in GenBank (Altschul et al. 1990).

From this process we recorded 17 strains of endophytes that, over subsequent BLAST analyses between 2017 and 2020, had top matches to C. prunicola (the holotype, represented by CBS 120875, sequence accession NR137037.1; Damm et al. 2010); C. endophytica and relatives (i.e., the strains described in Harrington et al. 2019: NC1642, 9094, and 9055); and a strain labelled as C. cephalothecoides (L821, accession KY064029.1; Han et al. 2017), reflecting the release of this sequence on 
Table 1. Taxon sampling, including proposed taxonomic placement, isolate codes, host taxa, regions of origin, isolation medium, storage period (the number of days between host tissue collection and fungal isolation, as described in Oita et al. 2021), and references for original isolation of each strain. Species names are based on the present study (see Fig. 1) and Harrington et al. (2019). Media abbreviations: MEA, malt extract agar; PDA, potato dextrose agar; SDA, Sabouraud's dextrose agar, CCA, cellulose medium; MYA, molasses yeast medium. References: 1, Hoffman \& Arnold 2008; 2, Damm et al. 2010; 3, U'Ren et al. 2012; 4, U'Ren et al. 2019; 5, Oita et al. 2021. NA, not available.

\begin{tabular}{|c|c|c|c|c|c|c|}
\hline Species & Isolate & Host & Region & Isolation medium & Storage period & Ref. \\
\hline C. endophytica & $9055^{*}$ & Platycladus orientalis & Tucson, AZ & MEA & 1 & 1 \\
\hline C. endophytica & 9069 & Platycladus orientalis & Tucson, AZ & MEA & 1 & 1 \\
\hline C. endophytica & 9093 & Platycladus orientalis & Tucson, AZ & MEA & 1 & 1 \\
\hline C. endophytica & 9094 & Platycladus orientalis & Tucson, AZ & MEA & 1 & 1 \\
\hline C. endophytica & FL0922 & Cladonia subradiata & Archbold, FL & MEA & 1 & 3 \\
\hline C. cf. endophytica & $\mathrm{NC} 1642$ & Sticta beauvoisii & Highlands, NC & MEA & 1 & 3 \\
\hline C. lutea & CBS121445 & Prunus salicina & South Africa & PDA & NA & 3 \\
\hline C. lutea & ER0164 & Parmeliaceae sp. & Russia & MEA & 7 & 2 \\
\hline C. lutea & SO10801 & Juniperus virginiana & Durham, NC & PDA & 10 & 5 \\
\hline C. lutea & SO06842 & Juniperus virginiana & Durham, NC & SDA & 1 & 5 \\
\hline C. lutea & SO06941 & Juniperus virginiana & Durham, NC & PDA & 1 & 5 \\
\hline C. lutea & SO08227 & Juniperus virginiana & Durham, NC & $\mathrm{CCA}$ & 5 & 5 \\
\hline C. lutea & SO08250 & Juniperus virginiana & Durham, NC & SDA & 5 & 5 \\
\hline C. lutea & SO08450 & Juniperus virginiana & Durham, NC & MYA & 5 & 5 \\
\hline C. lutea & SO08474 & Juniperus virginiana & Durham, NC & MYA & 5 & 5 \\
\hline C. lutea & SO09260 & Juniperus virginiana & Durham, NC & MYA & 5 & 5 \\
\hline C. palaoa & NC0604 & Hурпит sp. & Highlands, NC & MEA & 1 & 3 \\
\hline C. palaoa & SO06001 & Thelypteris sp. & Durham, NC & PDA & 1 & 5 \\
\hline C. prunicola & CBS120875 & Prunus armeniaca & South Africa & PDA & NA & 2 \\
\hline C. prunicola & IL0344 & Umbilicaria mammulata & Ives Lake, MI & MEA & 1 & 4 \\
\hline C. prunicola & SO07090 & Juniperus virginiana & Durham, NC & MYA & 1 & 5 \\
\hline C. prunicola & SO09965 & Juniperus virginiana & Durham, NC & SDA & 10 & 5 \\
\hline Outgroup & FL0068 & Pinus elliottii & Archbold, FL & MEA & 1 & 3 \\
\hline Outgroup & FL1226 & Cladonia subradiata & Archbold, FL & MEA & 1 & 3 \\
\hline
\end{tabular}

GenBank in fall of 2017. As discussed by Harrington et al. (2019), neither the type specimen of C. cephalothecoides nor the isolate with a ITS rDNA sequence labeled as C. cephalothecoides is available publicly. The authors who released the ITS rDNA sequence purporting to represent C. cephalothecoides did not examine type material, and the key characters of the species as originally described are not represented clearly in images published with the species description. Thus the identity of the sequence representing ' $C$. cephalothecoides' cannot be verified, and strains representing this species cannot be obtained for morphological characterization nor integrated readily into multilocus analyses for robust identification. We therefore focused on available strains in the C. endophytica / C. prunicola clade, including the focal endophytes for multilocus sequencing and morphological characterization. We note that the ITS rDNA sequence for ' $C$. cephalothecoides' was not identical to that of any of the isolates considered here, and that the morphological description of that species is not consistent with any of the species considered herein.

\section{Multilocus sequencing}

Cultures were revived from living vouchers in sterile water by plating on $2 \%$ MEA in $60 \mathrm{~mm}$ Petri dishes. These cultures were used for DNA extraction and to generate new cultures that were used to characterize morphology.

We extracted total genomic DNA directly from living mycelium at the growing edge of cultures on $2 \%$ MEA. Methods followed Harrington et al. (2019). We used
PCR to amplify additional loci from the 17 endophytes to complement and extend the ITS rDNA data set (see Table 2 for primers, sequence lengths, and references): actin-related protein $3(A C T)$, glyceraldehyde-3-phosphate dehydrogenase $(G P D)$, the RNA polymerase II second largest subunit (RPB2), and partial translation elongation factor 1-alpha (TEF-1a). We also amplified $A C T, G P D$, and $R P B 2$ for representative strains studied by Harrington et al. (2019): NC1642, 9055, 9094, the ex-type and ex-paratype strains of $C$. prunicola as determined by Damm et al. (2010) (CBS 120875 and CBS 121445 , respectively), and two outgroup strains (FL0068, FL1226). We obtained ITS rDNA and TEF-1 $a$ sequences for seven strains from GenBank, published previously by Harrington et al. (2019). Accession numbers are listed in Table 3.

Each PCR volume $(20 \mu \mathrm{L})$ consisted of $10 \mu \mathrm{L}$ of REDTaq ReadyMix (Sigma-Aldrich, St. Louis, MO, USA), $0.8 \mu \mathrm{L}$ of each $10 \mu \mathrm{M}$ primer, $1.3 \mu \mathrm{L}$ of $15 \mathrm{mg} / \mathrm{mL}$ BSA (New England BioLabs, Ipswitch, MA, USA), 3.1 $\mu \mathrm{L}$ of PCR water, and $4 \mu \mathrm{L}$ of DNA extract. Cycling parameters followed Harrington et al. (2019), typically with $54^{\circ} \mathrm{C}$ as the annealing temperature. We confirmed amplification with gel electrophoresis (1\% agarose gel with SYBR Green I; Molecular Probes, Invitrogen; Carlsbad, CA, USA). We cleaned PCR products by adding $1 \mu \mathrm{L}$ of ExoSAP-IT (Affymetrix, Santa Clara, CA, USA) to each reaction volume, then incubating for $60 \mathrm{~min}$ at $37^{\circ} \mathrm{C}$ and $15 \mathrm{~min}$ at $80^{\circ} \mathrm{C}$. We sequenced cleaned products with the original primers $(5 \mu \mathrm{M})$ on an Applied Biosystems 
Table 2. Loci amplified in addition to ITS rDNA (451 base pairs, bp; 23 parsimony-informative characters), including actin-related protein $3(A C T, 538 \mathrm{bp} ; 47$ variable and parsimony-informative characters), glyceraldehyde-3-phosphate dehydrogenase (GPD, 519 bp; 61 variable characters, of which 48 are parsimony-informative), the RNA polymerase II second largest subunit (RPB2, 1058 bp; 21 variable characters, of which 14 are parsimony-informative), and partial translation elongation factor 1-alpha (TEF-1a, 814 bp; 45 variable characters, of which 24 are parsimony-informative). Primers designed for the present study were developed by PI based on data from Harrington et al. (2019).

\begin{tabular}{l|l|l|l}
\hline Locus & \multicolumn{1}{|c|}{ Primer } & \multicolumn{1}{|c}{ Sequence } & \multicolumn{1}{c}{ References } \\
\hline$A C T$ & Arp3_446f & TGGGCCAGGTTCGTTACTTC & Present study \\
$A C T$ & Arp3_1344r & GTTCTTGTAAAGCCCGCGAC & Present study \\
$G P D$ & GPD_128f & TAAGTAGCCCCGAGACTGCT & Present study \\
$G P D$ & EF_648r & CTTCTGGGTGGCAGTGTAGG & Present study \\
$R P B 2$ & RPB2_57f & CAAGGGCTGGAAGAAGGAGG & Present study \\
$R P B 2$ & RPB2_983r & GAACTTGCAGGCAATGTGGG & Present study \\
$T E F-1 a$ & 983f & GCYCCYGGHCAYCGTGAYTTYAT & Rehner \& Buckley 2005; del Olmo Ruiz 2012 \\
$T E F-1 a$ & 2218R & ATGACACCRACRGCRACRGTYTG & Rehner \& Buckley 2005; del Olmo Ruiz 2012 \\
\hline
\end{tabular}

Table 3. GenBank accession numbers for sequence data. ${ }^{*}=$ from Harrington et al. 2019. Species names are based on the present study (see Fig. 1, Table 1) and Harrington et al. (2019). ND, not determined.

\begin{tabular}{|c|c|c|c|c|c|c|}
\hline Species & Isolate & ITSrDNA & $A C T$ & $G P D$ & $R P B 2$ & $T E F-1 a$ \\
\hline C. endophytica & 9055 & MK614056 & MZ241161 & MZ241194 & MZ241216 & MK693165* \\
\hline C. endophytica & 9069 & MZ241144 & MZ241162 & MZ241195 & MZ241217 & MZ241185 \\
\hline C. endophytica & 9093 & MZ241145 & MZ241163 & MZ241196 & MZ241218 & MZ241186 \\
\hline C. endophytica & 9094 & EF420005* & MZ241164 & MZ241197 & MZ241219 & MK693159* \\
\hline C. endophytica & FL0922 & MZ241147 & MZ241169 & MZ241202 & MZ241223 & ND \\
\hline C. cf. endophytica & $\mathrm{NC} 1642$ & JQ761997* & MZ241173 & MZ241205 & MZ241226 & MK693164* \\
\hline C. lutea & CBS121445 & GQ154541* & MZ241166 & MZ241199 & MZ241221 & MK693161* \\
\hline C. lutea & ER0164 & MZ241146 & MZ241167 & MZ241200 & MZ241222 & ND \\
\hline C. lutea & SO06842 & MZ241151 & MZ241175 & MZ241207 & MZ241228 & ND \\
\hline C. lutea & SO06941 & MZ241152 & MZ241176 & MZ241208 & MZ241229 & MZ241190 \\
\hline C. lutea & SO08227 & MZ241154 & MZ241178 & MZ241210 & MZ241231 & MZ241191 \\
\hline C. lutea & SO08250 & MZ241155 & MZ241179 & MZ241211 & MZ241232 & ND \\
\hline C. lutea & SO08450 & MZ241156 & MZ241180 & ND & MZ241233 & ND \\
\hline C. lutea. & SO08474 & MZ241157 & MZ241181 & MZ241212 & MZ241234 & MZ241192 \\
\hline C. lutea & SO09260 & MZ241158 & MZ241182 & MZ241213 & MZ241235 & ND \\
\hline C. lutea & SO10801 & MZ241160 & MZ241184 & MZ241215 & MZ241237 & MZ241193 \\
\hline C. palaoa & NC0604 & MZ241149 & MZ241172 & MZ241204 & MZ241225 & MZ241188 \\
\hline C. palaoa & SO06001 & MZ241150 & MZ241174 & MZ241206 & MZ241227 & MZ241189 \\
\hline C. prunicola & CBS120875 & NR137037* & MZ241165 & MZ241198 & MZ241220 & MK693162* \\
\hline C. prunicola & IL0344 & MZ241148 & MZ241171 & ND & MZ241224 & MZ241187 \\
\hline C. prunicola & SO07090 & MZ241153 & MZ241177 & MZ241209 & MZ241230 & ND \\
\hline C. prunicola & SO09965 & MZ241159 & MZ241183 & MZ241214 & MZ241236 & ND \\
\hline Outgroup & FL0068 & JQ759927* & MZ241168 & MZ241201 & ND & MK693157* \\
\hline Outgroup & FL1226 & JQ760841* & MZ241170 & MZ241203 & ND & MK693158* \\
\hline
\end{tabular}

$373 x l$ (Foster City, CA, USA) at the University of Arizona Genetics Core (UAGC). We assembled contigs and evaluated their quality with phred/phrap (Ewing et al. 1998) in Mesquite v2.75 (Maddison \& Maddison 2017), with manual confirmation of base calls in Sequencher v.4.5 (Gene Codes, Ann Arbor, MI, USA). All newly generated data were submitted to GenBank (Table 3).

\section{Phylogenetic analyses}

Data for each locus were aligned in MUSCLE (Edgar 2004) and trimmed to consistent starting and ending points prior to analysis via maximum likelihood (ML) in GARLI v0.95 (Zwickl 2006) and parsimony (MP) in PAUP* v4.0a build 168 . We used the GTR $+\mathrm{I}+\mathrm{G}$ model for the former, and evaluated topological support in both approaches via 1000 bootstrap replicates. We analyzed each locus separately and then analyzed the data as a concatenated data set that comprised 24 terminals, of which two were outgroups, with each terminal represented by at least four loci. No conflict was observed when single-locus trees were compared (data not shown; for single-locus topologies see Figures S1-S5). The final alignment was trimmed to consistent starting and ending points, for a total length of 3370 characters. Of these, 198 were variable and 156 were parsimony-informative. Information regarding the number parsimony-informative characters for each locus is presented in Table 2. Alignments from this study are archived at TreeBASE (accession 28547).

\section{Morphological evaluation}

We observed cultures of all strains on standard media (MEA and PDA) after incubation for 3 months at $22^{\circ} \mathrm{C}$ 
Table 4. Growth and macroscopic characteristics of focal strains on standard media after 1 month at $22^{\circ} \mathrm{C}$ with natural light-dark conditions. Species names are based on the present study (see Fig. 1) and Harrington et al. (2019). Colony characteristics were variable within and between species in terms of growth rate and colony pigmentation, and as indicated by Harrington et al. (2019), growth on these media was generally not diagnostic of species boundaries without additional information (see Table 5 and Discussion). Growth rates on each medium were relative to the fastest and slowest growth in the study.

\begin{tabular}{l|l|l|l|l|l}
\hline Species & \multicolumn{1}{|c|}{ Isolate } & \multicolumn{1}{|c|}{ PDA colony } & \multicolumn{1}{|c}{ PDA below } & \multicolumn{1}{c}{ MEA colony } & \multicolumn{1}{c}{ MEA below } \\
\hline C. endophytica & 9055 & Moderate, white-pink to white & Pale orange & White-pink & Pale orange \\
C. endophytica & 9094 & Slow, white-orange to white & Orange-white & White-orange & Orange-white \\
C. endophytica & FL0922 & Moderate, white & White & White & Salmon \\
C. cf. endophytica & NC1642 & Rapid, white-yellow & Light orange & Pastel red & Pastel red \\
C. lutea & CBS121445 & Moderate, white-orange & Light orange & White-pink & Beige \\
C. palaoa & NC0604 & Moderate, white-yellow & White-yellow & Pale pink & Pink-orange \\
C. prunicola & CBS120875 & Rapid, beige-orange & Light pink-orange & Pastel red & Pink-orange \\
Outgroup & FL0068 & Rapid, beige-orange & Orange-white & Pale orange & Pale orange \\
Outgroup & FL1226 & Moderate, white-pink & White-pink & Pinkish beige & Pink \\
\hline
\end{tabular}

Table 5. Growth and macroscopic characteristics of focal strains on diverse media at $22^{\circ} \mathrm{C}$ with natural light-dark conditions. Positive indicates bioactivity for a given assay plate. All isolates demonstrated chitinase activity, and all grew readily on ligninase and cellulase media, as well as on thrice-autoclaved pine needles. Fertile structures typically were observed after $\sim 6$ months in culture, including $\sim 3$ months of storage at $4^{\circ} \mathrm{C}$. Taken together, growth characteristics on these selective media were relatively more informative of species boundaries than standard media (Table 4). Names are based on the present study (see Fig. 1) and Harrington et al. (2019). Growth rates on each medium were relative to the fastest and slowest growth in the study.

\begin{tabular}{l|l|l|l|l|l}
\hline Species & \multicolumn{1}{|c|}{ Isolate } & \multicolumn{1}{c|}{ Chitinase } & \multicolumn{1}{c}{ Ligninase } & \multicolumn{1}{c}{ Cellulase } & \multicolumn{1}{c}{ Pine needles } \\
\hline C. endophytica & 9055 & Positive & Slow, beige & Rapid, white & Infertile perithecia \\
C. endophytica & 9094 & Positive & Slow, beige & Rapid, white & Infertile perithecia \\
C. endophytica & FL0922 & Positive & Slow, beige & Rapid, white & Infertile perithecia \\
C. cf. endophytica & NC1642 & Positive & Slow, beige & Rapid, white & Fertile \\
C. lutea & CBS121445 & Positive & Slow, beige & Moderate, beige & Fertile \\
C. palaoa & NC0604 & Positive & Moderate, colorless & Rapid, white & Fertile \\
C. prunicola & CBS120875 & Positive & Slow, white-yellow & Rapid, white-yellow & Fertile \\
Outgroup & FL0068 & Positive & Rapid, colorless & Slow, colorless & Sterile \\
Outgroup & FL1226 & Positive & Rapid, colorless & Moderate, beige & Sterile \\
\hline
\end{tabular}

with natural light-dark cycles. Given the morphological variability of these strains on standard media, we also evaluated growth on chitinase medium (Agrawal \& Kotasthane 2012), ligninase medium, containing indulin (Gazis et al. 2012), cellulase medium, containing cellulose (Gazis et al. 2012), and thrice autoclaved pine needles on water agar (see Harrington et al. 2019). Macroscopic characteristics are shown in Table 4 and Table 5. Microscopic characters were compared against references for the previously described members of the clade: C. prunicola (Damm et al. 2010) and C. endophytica (Harrington et al. 2019), as well as C. cephalothecoides (Kamiya et al. 1995). Micromorphological features were imaged with a Leica DM4000B compound microscope. A minimum of 25 conidia and conidiogenous cells per isolate were measured with ImageJ and a stage micrometer for each focal isolate.

\section{Results}

Phylogenetic analyses of endophytes within the C. endophytica / C. prunicola clade reveal four distinct lineages based on maximum likelihood and parsimony analyses of the concatenated dataset (ITS rDNA, $A C T, G P D, T E F-1 a$, and $R P B 2$ ) (Fig. 1). Among these, $A C T$ and $G D P$ were especially informative (see details in Table 2). The four lineages are supported robustly and are here proposed to reflect four species, as described below. The results presented here also expand current knowledge of the hostand geographic ranges of each previously described taxon. We describe each below, focusing first on the presently recognized species.

First, the topology inferred here expands the known geographic range of $C$. endophytica to include Florida as well as its previously recognized distribution in Arizona, USA. The analysis highlights that this species occurs not only in conifers (Cupressaceae, Harrington et al. 2019) but also in healthy thalli of lichens (here, Cladonia subradiata; Table 1). As in previous work (Harrington et al. 2019) we observe the affiliation of NC1642 with strains identified here as C. endophytica (Fig. 1). NC1642 is morphologically distinct from the strains formally recognized as C. endophytica (Table 4, Table 5) and differs by its production of fertile structures on pine needles, unlike the strains described as $C$. endophytica (see Harrington et al. 2019). We consider it closely allied with C. endophytica.

Second, the topology expands the geographic and host ranges known for C. prunicola (as defined by the ex-type, CBS 120875). This strain, first identified from necrotic wood of a non-native, angiosperm tree cultivated in South Africa (Table 1), forms a well-supported clade with endophytes of hosts native to North America, including Juniperus virginiana (Cupressaceae) in North Carolina and the lichen Umbilicaria mammulata from 


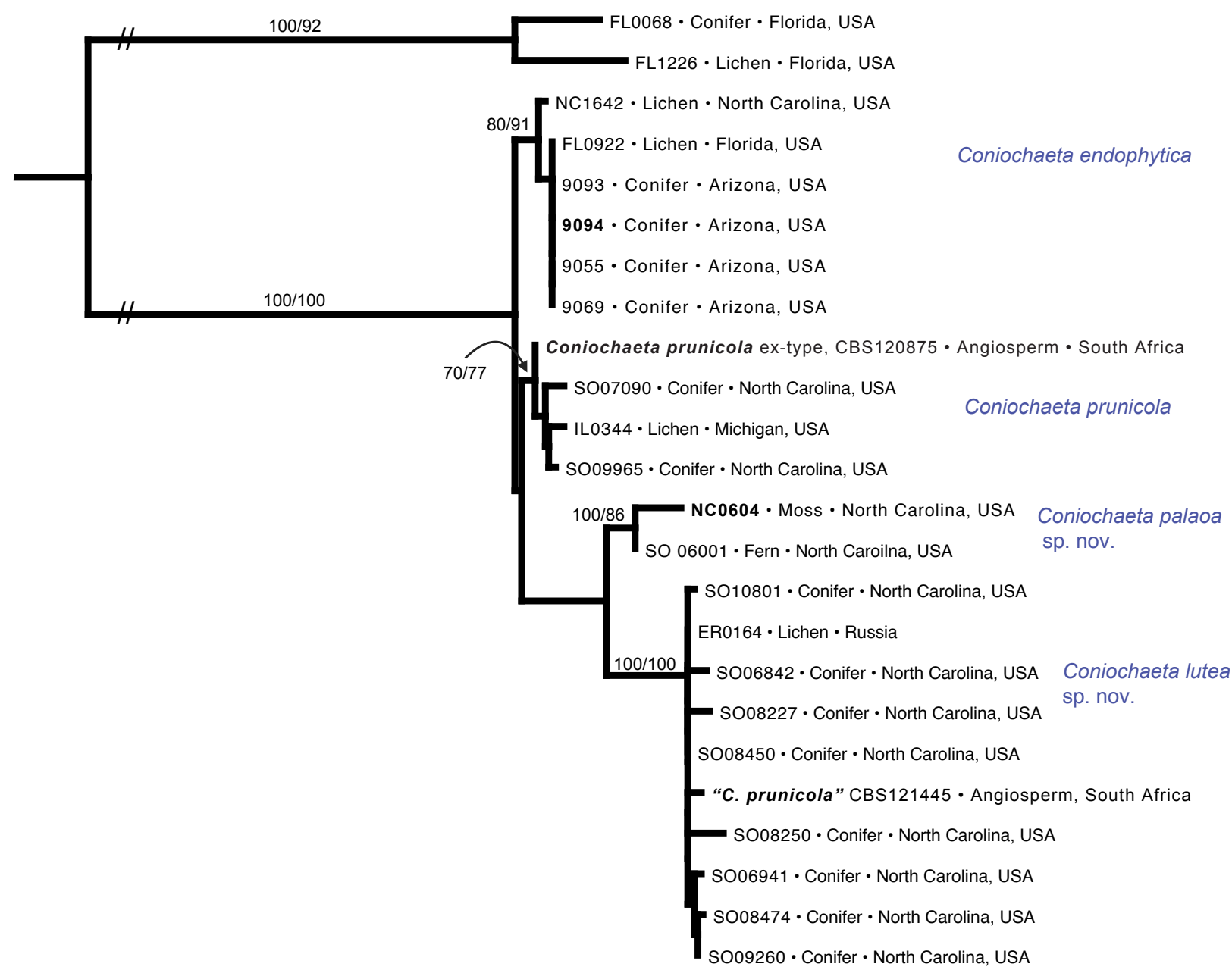

Figure 1. Taxonomic placement and resolution of main groups within the C. endophytica / C. prunicola clade, as inferred via maximum likelihood (ML). Topologies inferred via ML and maximum parsimony (MP) were identical. Support values $\geq 70 \%$ from 1000 bootstrap replicates shown as values above nodes (MP/ML). The type strain for each species is shown in bold. Host information summarizes data from Table 1.

rock faces at the boreal / temperate forest interface in the Upper Peninsula of Michigan (Table 1). Together the wide geographic- and host ranges of this species in North America, and its occurrence in native North American hosts, suggest that its occurrence under cultivation conditions in South Africa may reflect an introduction rather than native occurrence there.

Third, the analysis reveals that the ex-paratype for C. prunicola (CBS 121445) forms a distinct clade with endophytes from North Carolina and Russia, and is not reconstructed as conspecific with the ex-type strain of that species (i.e., CBS 120875). The strain represented by CBS 121445 was isolated originally from an angiosperm tree cultivated in South Africa (Damm et al. 2010). The clade containing this strain is here shown to include endophytes from a conifer ( $J$. virginiana) and a lichen in the Parmeliaceae (Fig. 1; Table 1). Our results support segregation of this clade from C. prunicola as defined by the ex-type (i.e., C. prunicola sensu stricto), and here we propose to designate this lineage as $C$. lutea, as described below. This result corroborates the previous observation that the two strains designated as $C$. prunicola (i.e, the ex-type and paratype) differ in several characteristics, including spore size, growth rate on certain media, and macroscopic characters (see Harrington et al. 2019). Here we show the species that includes CBS 121445 (i.e., C. lutea) has distinctive colony morphology and growth characteristics on several media (Table 4, Table 5) relative to the C. prunicola ex-type and related endophytes. The wide geographic distribution of this species, which includes hosts on three continents, remains to be explored.

Fourth, the analysis identifies an additional new species within the C. endophytica / C. prunicola clade, here proposed as Coniochaeta palaoa. Coniochaeta palaoa was observed as an endophyte of a moss and a fern in the Appalachian highlands and piedmont forests of North Carolina, respectively. A suite of morphological characteristics at the whole-colony level, as observed for strains on multiple media, underscores the distinctiveness of this species, which is further supported by microscopic characters (see below).

Finally, we note that inclusion of the ITS rDNA sequence labeled as 'C. cephalothecoides' does not change these relationships (data not shown). The position of this putative 'C. cephalothecoides' isolate remains uncertain as described above.

\section{Morphological characterization}

Colony coloration from above and below varied within and among species on both MEA and PDA (Table 4). In 
general, the strains considered here ranged in main colony color on PDA and MEA from white to pink, orange, red, or yellow, and in reverse colony colors from orange-white and pale pink to yellowish (Table 3). All strains lacked colored exudates in growth media. Isolates ranged from copious to lacking in terms of aerial mycelium, with variation within and among species. All isolates demonstrated chitinase activity in vitro and grew readily, albeit at different relative growth rates and with distinct colony characteristics, on cellulose- and indulin-based media (Table 5).

All isolates considered here featured discrete, intercalary phialides. They lacked chlamydospores and had oblong to occasionally spheroid or ovoid conidia that varied in size and shape within and among species (Fig. 2). Harrington et al. (2019) noted that the ex-type and paratype strains then considered to represent $C$. prunicola (i.e., CBS 120875, CBS 121445) differed from each other in conidial length and width. Even though conidial size and shape appear to be plastic in this group, this observation is broadly consistent with differentiation of these two strains into different species as described here (Fig. 1). Damm et al. (2010) reported that $C$. prunicola has conidia (2.5)3.5-6.0(8.0) × 1.0-2.0(3.0) $\mu \mathrm{m}$. Those of the ex-paratype (hereafter, C. lutea) measured (2.0)2.3-4.2(5.0) $\times$ (0.6)0.9-1.8(2.0) $\mu \mathrm{m}$ as reported by Harrington et al. (2019) (but see the taxonomic section for additional measurements and discussion, as larger conidia were observed in the present study). Conidia of C. endophytica (9094) were reported as (2.5)3.1-3.4(4.4) × (1.3)1.6-1.8(2.4) $\mu \mathrm{m}$ (Harrington et al. 2019). Conidia of C. palaoa (NC0604) measured (3.1)3.6-4.7(5.3) × (1.0)1.5-1.9(2.4) $\mu \mathrm{m}$. We observed conidia of $C$. palaoa to present as both relatively spherical and oblong, but they consistently fell within the range of values presented here.

As noted by Harrington et al. (2019), conidiophores in C. endophytica are more ampulliform and more likely to be linearly extended than those of $C$. prunicola (ex-type; Damm et al. 2010). Damm et al. (2010) also noted distinctive collarettes in C. prunicola (ex-type). We observed only indistinct collarettes in C. endophytica, C. lutea, and C. palaoa (Fig. 2). Conidiogenous cells of C. palaoa
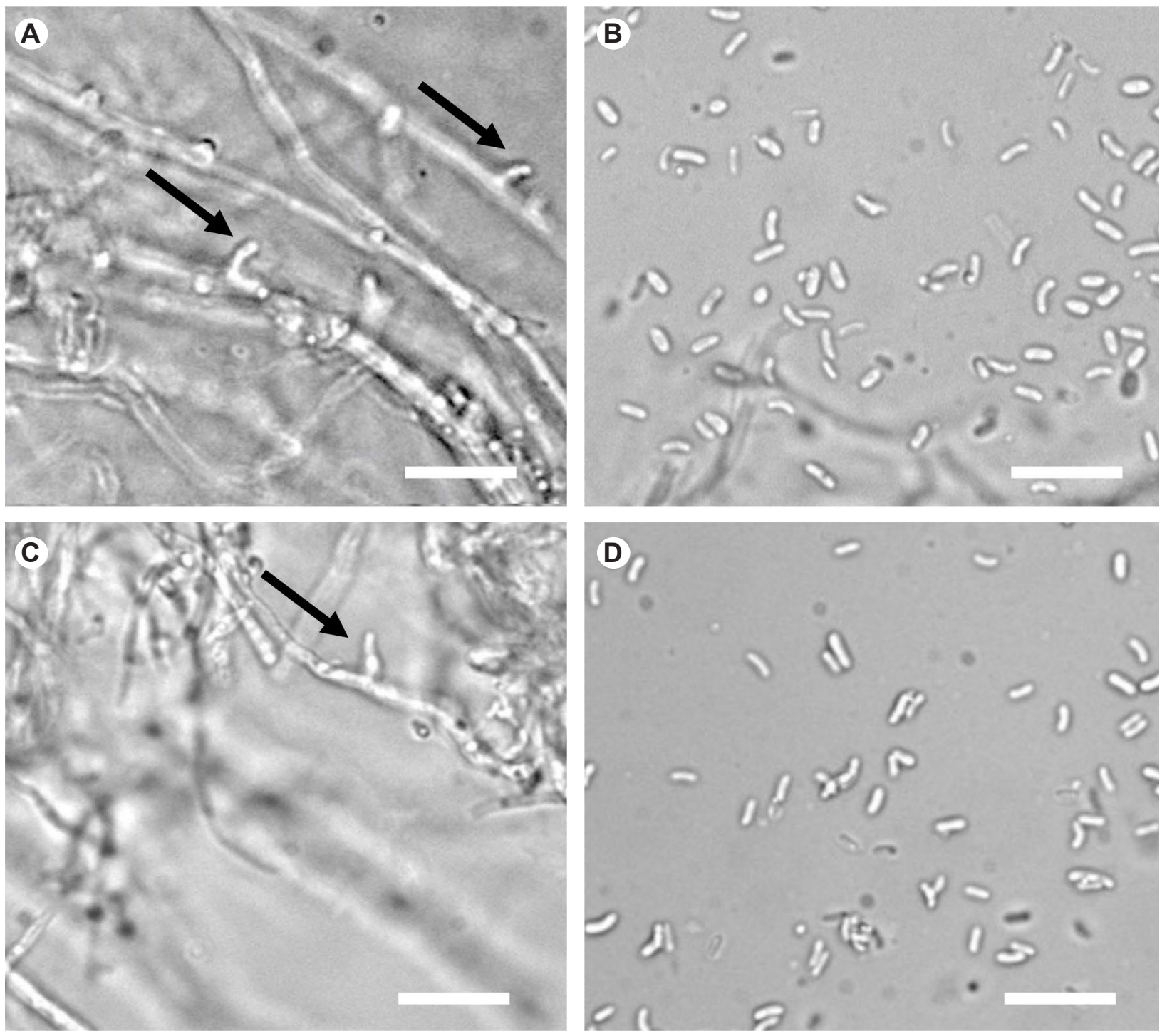

Figure 2. Conidiophores and conidia: A-B - Coniochaeta lutea (ARIZ-AEAexCBS 121445); C-D - Coniochaeta palaoa (ARIZ-AEANC0604). Arrows indicate conidiogenous cells, which are more linear and bent at the tips in C. lutea, and relatively more ampulliform in C. palaoa. Conidia are similar in the two species. Scale $=10 \mu \mathrm{m}$. 

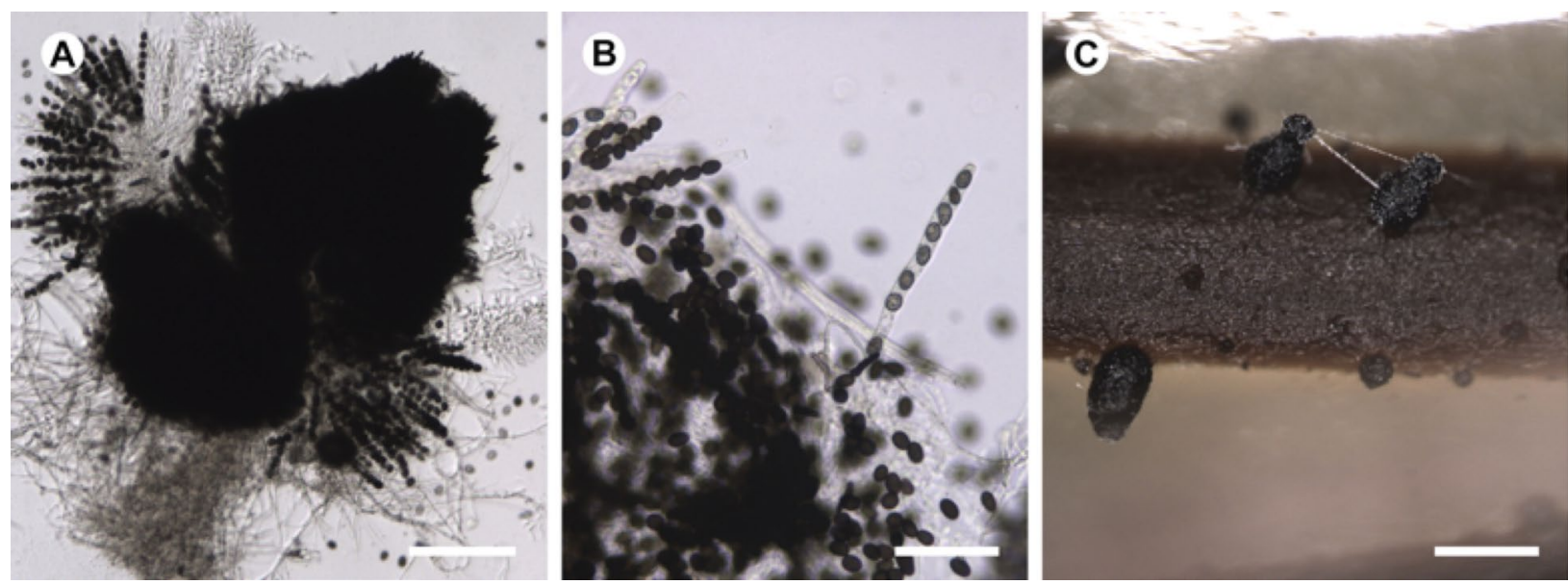

Figure 3. Coniochaeta lutea (ARIZ-AEAexCBS 121445). A - mature ascoma with asci and ascospores, with hyaline paraphyses visible, and dark setae visible at the top part of the perithecium; B - asci and ascospores; C - mature perithecia on thrice-autoclaved pine needle. Scales: $\mathrm{A}=100 \mu \mathrm{m} ; \mathrm{B}=50 \mu \mathrm{m} ; \mathrm{C}=200 \mu \mathrm{m}$.

(NC0604) are often bent at the ends or long in shape (Fig. 2). Those of C. lutea (CBS 121445) are similar to those of C. palaoa (Fig. 2).

Isolates classified as C. lutea (Figs 2-3; Fig. S6), C. palaoa (Figs 2, 4; Fig. S6), and C. prunicola (per the ex-type, Damm et al. 2010; Fig. 5) produced fertile structures on thrice-autoclaved pine needles, but $C$. endophytica did not. Those fertile structures were observed six months after pine needles were inoculated. During that period, strains were allowed to grow for approximately two months at room temperature with natural light-dark cycles, and then refrigerated in darkness at $4^{\circ} \mathrm{C}$ for three months, followed by one month at room temperature. We did not observe fertile structures on any other media. In all cases, perithecia were dark brown, contrasting with the black coloration reported by Kamiya et al. (1995) for $C$. cephalothecoides. Ascomata and ascospores are described for C. lutea and C. palaoa in the taxonomic descriptions below, wherein we compare them to one another and to those of $C$. prunicola (ex-type), and those of $C$. cf. endophytica (NC1642), as the strains identified unequivocally as $C$. endophytica have not yet been observed to produce sexual structures in culture.

\section{Taxonomy}

Coniochaeta lutea A.E. Arnold, and A.H. Harrington, sp. nov.

\section{MycoBank MB839428}

Type: South Africa, Limpopo Province, Mookgopong, on necrotic wood of Prunus salicina, Aug. 2004, U. Damm ARIZ-AEAexCBS 121445 - holotype, preserved in a metabolically inactive state, ex-type culture deposited in the culture collection of the Westerdijk Institute (CBS-KNAW) as CBS 121445 (originally deposited as the paratype of C. prunicola Damm \& Crous).

Description. Colonies on PDA attained a diameter of $\sim 60 \mathrm{~mm}$ after 30 days at $22^{\circ} \mathrm{C}$, predominantly white to light orange in aspect (5A2), with sparse and woolly, white aerial hyphae (5A1) and the colony bearing light orange coloration (5A3) at the colony center, especially visible from below. Colonies on MEA were similar but had a pinkish white aspect at the colony center (72A), visible from above. After cold storage, coloration of the center of the colonies on PDA was a strong pastel yellow (3A3) with aerial hyphae remaining white. No diffusable pigments were observed. Chitinase positive; slow and beige growth on ligninase assay medium, and moderate and beige growth with elongated aerial hyphae on cellualse assay medium. Vegetative hyphae hyaline, without chlamydospores, 1-4 $\mu \mathrm{m}$ wide. Conidiogenous cells phialidic, ampulliform, with a constricted base, arising directly from hyphae, terminal. Conidia hyaline, single-celled, smooth-walled, bacilliform to allantoid, abundant on all media examined, (3.1)3.7-4.1(5.4) $\mu \mathrm{m}$ $\times(1.0) 1.3-1.6(1.8) \mu \mathrm{m}$. Ascomata: fertile perithecia formed on autoclaved pine needles and in minimal media surrounding pine needles; solitary or rarely aggregated, superficial on the substrate but occasionally immersed; globose but slightly elongated or pear-shaped at maturity, with a short neck producing a round mass of ascospores; diameter, (181)201-267(294) $\mu \mathrm{m}$, averaging $232 \mu \mathrm{m}$; length, (338)389-432(487) $\mu \mathrm{m}$, averaging $411 \mu \mathrm{m}$, including the neck and spore mass; peridium dark brown, pseudoparynchymatous, densely covered in dark brown, short, unbranched, coarse setae. No ascomata were observed on PDA, MEA, or the media described in Table 5. Asci cylindrical, unitunicate, containing 8 ascospores, growing from central point at base of ascoma alongside hyaline paraphyses. Ascospores at maturity dark brown, uniseriate, single celled, smooth-walled, with a longitudinal germ slit; ovoid and broadly ellipsoidal; (7.2)8.6-9.7(10.2) $\mu \mathrm{m}$ $\times(4.7) 5.3-6.4(7.2) \mu \mathrm{m}$; when immature, smooth-walled, beige, translucent with granular contents, saucer-shaped. See Figs 2-3, and Fig. S6.

Etymology. Referring to its distinctive yellow aspect on PDA after three months of cold storage, reminiscent of spring after an arduous season.

Notes. All isolates discussed in this paper were processed at the same time and under the same conditions, including the three months of cold storage necessitated 
by a research closure during the COVID-19 pandemic. All isolates representing C. prunicola, $C$. endophytica and C. palaoa, lacked the yellow coloration that was present after cold storage in all strains designated here as $C$. lutea. Harrington et al. (2019) stated that conidia of CBS 121445 measured (2.0)2.3-4.2(5.0) × (0.6)0.9-1.8(2.0) $\mu \mathrm{m}$, but here we record larger conidia (above), in line with the observation that conidial dimensions seem somewhat plastic in this clade as a whole (see also Harrington et al. 2019 for evidence of conidial size variation in C. endophytica). The ascospore dimensions reported here are smaller than those reported for $C$. cephalothecoides by Kamiya et al. (1995) and somewhat smaller than those reported for C. prunicola by Damm et al. (2010) and C. cf. endophytica (NC1642) by Harrington et al. (2019). We did not observe ascomata formation on PDA, as reported for C. prunicola by Damm et al. (2010), nor on MEA or PDA as reported for NC1642 by Harrington et al. (2019). The abundant perithecia of $C$. lutea, occurring superficially on pine needles and on or semi-immersed in the medium near pine needles, were smaller than those reported by Kamiya et al. (1995) for C. cephalothecoides on culture media, smaller than those observed here for C. palaoa under the same conditions and handled concurrently, and similar in size to those reported by Damm et al. (2010) for C. prunicola and Harrington et al. (2019) for NC1642. Harrington et al. (2019) noted that perithecia of NC1642 feature a smaller, blunter neck than those of the ex-type culture of $C$. prunicola, and here we observe that the perithecial neck of $C$. lutea is even less elongate and more blunt than in NC1642 (see Fig. 3d in Harrington et al. 2019). The extensive hairs noted on the perithecia of $C$. cephalothecoides by Kamiya et al. (1995) were not observed in C. lutea.

Specimens examined. USA, North Carolina, Durham, strains SO10801, SO06842, SO08227, SO08450, SO08250, SO08474, SO09260, isolated by S. Oita from surface-sterilized, healthy foliage of Juniperus virginiana as described in Oita et al. (2021); and Russia, Khabarovsk, ER0164, isolated by Jana M. U'Ren from a surface-sterilized, symptomless thallus of a lichen in the Parmeliaceae (U'Ren et al. 2019).

Vouchers and data deposition. Living vouchers of the paratypes SO10801, SO06842, SO08227, SO08450, SO08250, SO08474, SO09260, and ER0164 are deposited in the publicly accessible culture collection of the Robert L. Gilbertson Mycological Herbarium at the University of Arizona (ARIZ; accession numbers match isolate numbers; see above for holotype information), with data available at MyCoPortal.org. Sequence data for ITS rDNA, $A C T$, GPD, RPB2, and TEF-1 $a$ are deposited in GenBank (Table 3).

Coniochaeta palaoa A.E. Arnold, A.H. Harrington, P. Inderbitzin, and V.K. Knight-Connoni, sp. nov.

\section{MycoBank MB839427}

Type: USA, North Carolina, Highlands Biological Station. Endophytic in healthy Hypnum sp., isolated from surface-sterilized, photosynthetic tissue by J.M. U'Ren ARIZ-AEANC0604 - holotype); preserved in a metabolically inactive state, ex-type culture deposited in the culture collection of the Westerdijk Institute (CBS-KNAW).

Description. Colonies on PDA attained a diameter of $\sim 55-65 \mathrm{~mm}$ after 30 days at $22^{\circ} \mathrm{C}$, predominantly white to pale yellow in aspect (2A3), with sparse and woolly aerial hyphae (2A1) and the colony bearing light yellow coloration (2A3) at the colony center, especially visible from below. Colonies on MEA were similar but had a pinkish white aspect at the colony center visible from above (82A), and a salmon aspect from below (6A4). Chitinase positive; moderate and colorless growth on ligninase assay medium; rapid and white growth on cellulase assay medium. No diffusible pigments were observed. Vegetative hyphae hyaline, without chlamydospores,
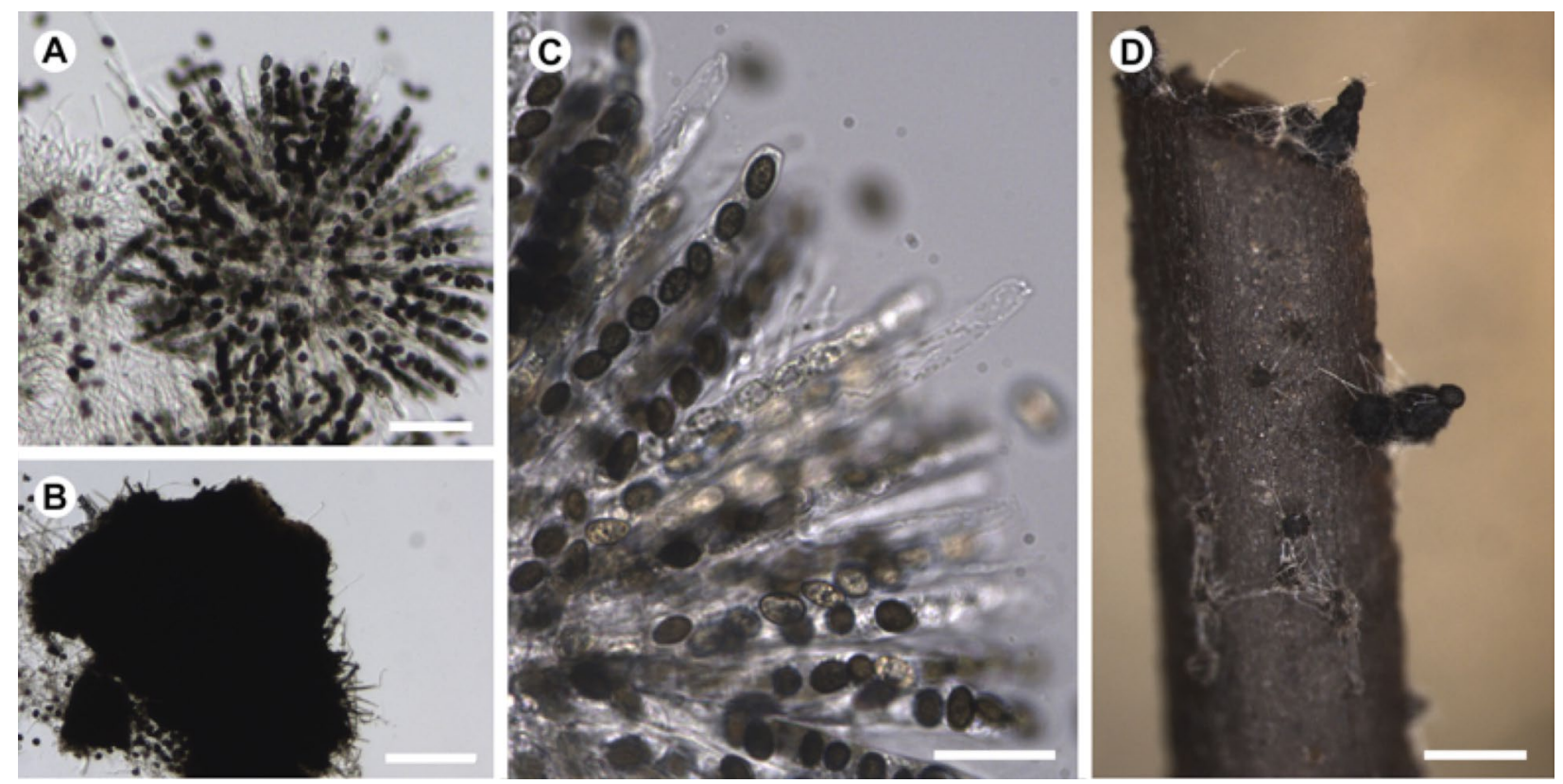

Figure 4. Coniochaeta palaoa (ARIZ-AEANC0604). A, C - mature asci and ascospores; B - mature perithecium; D - mature perithicia on thrice-autoclaved pine needle. Scales: $\mathrm{A}=50 \mu \mathrm{m} ; \mathrm{B}=100 \mu \mathrm{m} ; \mathrm{C}=25 \mu \mathrm{m} ; \mathrm{D}=200 \mu \mathrm{m}$. 

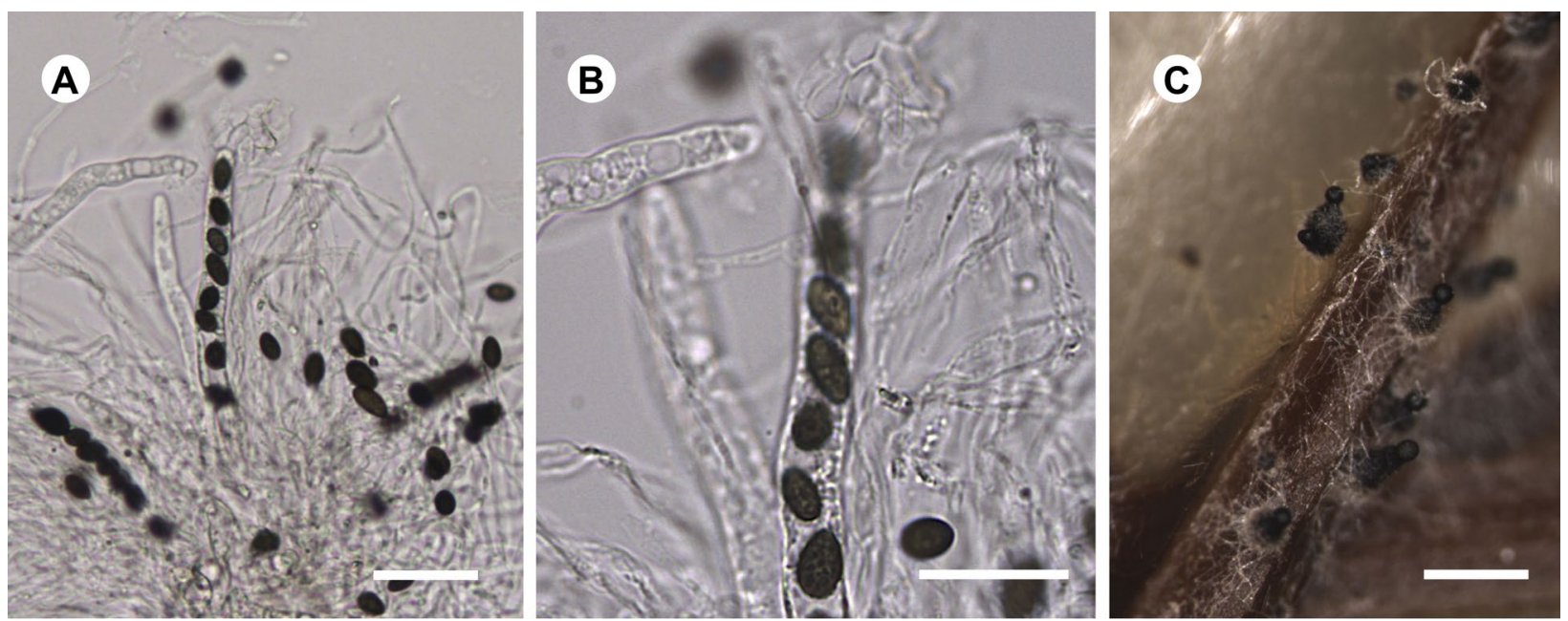

Figure 5. Coniochaeta prunicola, ex-type isolate CBS 120875. A-B - mature asci and ascospores; C - mature perithecia on thrice-autoclaved pine needle. Scales: $\mathrm{A}-\mathrm{B}=50 \mu \mathrm{m} ; \mathrm{C}=100 \mu \mathrm{m}$.

1-4 $\mu \mathrm{m}$ wide. Conidiogenous cells phialidic, ampulliform, with a constricted base, arising directly from hyphae, terminal. Conidia hyaline, single-celled, smooth-walled, bacilliform to allantoid, abundant on all media examined, (3.1)3.6-4.7(5.3) $\mu \mathrm{m} \times(1.0) 1.5-1.9(2.4) \mu \mathrm{m}$. Ascomata: fertile perithecia formed on autoclaved pine needles and in minimal media surrounding pine needle; solitary or rarely aggregated, superficial on the substrate but occasionally immersed; globose but slightly elongated or pear-shaped at maturity, with a short neck producing a round mass of ascospores; diameter, (206)232-268(286) $\mu \mathrm{m}$, averaging $249 \mu \mathrm{m}$; length, (324)398-462(488) $\mu \mathrm{m}$, averaging $423 \mu \mathrm{m}$, including the neck and mass of spores; peridium dark brown, pseudoparynchymatous, densely covered in dark brown, short, unbranched, coarse setae. Asci cylindrical, unitunicate; containing 8 ascospores; growing from central point at base of ascoma alongside hyaline paraphyses. Ascospores at maturity dark brown, uniseriate, single celled, smooth-walled, with a longitudinal germ slit, ovoid and broadly ellipsoidal, (7.7)8.6-9.7(10.7) $\mu \mathrm{m}$ $\times(4.7) 5.3-6.4(7.2) \mu \mathrm{m}$; when immature, smooth-walled, beige, translucent with granular contents, saucer-shaped. See Figs 2, 4, and Fig. S6.

Etymology. Named for its maize-colored growth in culture, its powdery and flour-like appearance on malt extract agar, and its growth-promoting activity in corn. The name is drawn from traditional Hawaiian, meaning maize or flour, linking these three elements in a single term while also speaking to the Pacific region of origin of the author who isolated the second strain (S. Oita).

Notes. Morphological characters that reliably distinguish this species are somewhat limited, but the maize color after cold storage was distinctive. Conidia of this species were variable in presentation, at times more elongate / ovoid and in other cases, more spherical, speaking to the apparent plasticity of conidial sizes in this clade as a whole. The ascospore dimensions reported here are smaller than those of $C$. cephalothecoides (Kamiya et al. 1995) and somewhat smaller than those of C. prunicola (Damm et al. 2010) and NC1642 (Harrington et al. 2019).
In contrast to $C$. prunicola and NC1642, we did not observe ascomata formation on PDA or MEA. Abundant perithecia were formed superficially on pine needles, and on or semi-immersed in the medium near pine needles; these were larger than those of C. lutea (above) and, in addition to being dark brown (vs. black, Kamiya et al. 1995), differed from those described for C. cephalothecoides by lacking extensive coverage by hairs. Among the isolates considered here, C. palaoa grew the fastest among the ingroup taxa on ligninase medium.

Specimens examined. USA, North Carolina, Durham, strain SO06001, isolated by S. Oita from surface-sterilized, healthy foliage of Juniperus virginiana as described in Oita et al. (2021).

Vouchers and data deposition. Living vouchers of the paratype SO06001 are deposited in the publicly accessible culture collection of the Robert L. Gilbertson Mycological Herbarium at the University of Arizona (ARIZ; accession numbers match isolate number; see above for holotype information), with data available at MyCoPortal.org. Sequence data for ITS rDNA, $A C T, G P D, R P B 2$, and TEF-1 $a$ are deposited in GenBank (Table 3).

\section{Discussion}

Fungal endophytes are widely recognized as highly diverse at a global scale (Rodriguez et al. 2009). Their under-representation in established culture collections raises the question: to what degree do endophytes represent novel species? This question can be difficult to answer in the absence of well-described specimens and associated DNA sequences, especially beyond the scope of only the barcode locus for fungi (ITS rDNA). In taxa such as Coniochaeta, closely related species often have highly similar ITS rDNA sequences, such that sequencing additional loci is necessary to differentiate species (see Harrington et al. 2019).

The present study expands the current view of a two-species clade within Coniochaeta (i.e., the clade containing $C$. prunicola, $C$. endophytica) by including 
endophytes from diverse hosts and substrates, with the result that the clade is expanded to include at least four species, and the host and geographic range of the known species are expanded. This study illustrates how endophytes and other collections from ecological studies, even if unnamed at the time of collection, can be integrated into later systematic studies if maintained as vouchers in formal collections (see also Carbone et al. 2016, 2018; Harrington et al. 2019).

The wide host- and geographic range of each species considered here suggests ecological lability within species of Coniochaeta and suggests that integrating them into studies of gene expression and symbiotic adaptation to hosts would be fruitful. While it is plausible that some of the distributions described here could be attributed to introduction into South Africa (e.g., of C. prunicola and $C$. lutea as described here), more rigorous analyses are needed to test this hypothesis. The distribution of C. lutea also appears to include the Russian Far East, with a three-continent spread that is surprising given how infrequently these species have been isolated. Notably, the wide geographic ranges of each species suggest that should endophytes in Coniochaeta be useful for modulating plant phenotypes (see Chen 2017), they may be useful under diverse environmental conditions and in different landscapes and hosts. The clade comprising C. endophytica, C. lutea, C. palaoa, and C. prunicola appears to be related closely to species such as $C$. africana, C. ligniaria and C. mutabilis (Friebes et al. 2016; Nasr et al. 2018), part of a lineage generally known from wood and decaying plant material. There is no evidence of mammalian pathogenicity among the strains discussed here.

In previous work on this clade, Harrington et al. (2019) used ITS rDNA and TEF-1a to distinguish C. prunicola and the species they described, $C$. endophytica. The present study further supports that division and provides evidence of robust resolving power in this clade by $A C T$, as well as the other loci considered here. Informative characters were especially common in $A C T$, with all loci providing additional resolving power (see Table 2). We anticipate that $A C T, G P D, R P B 2$, and TEF-1a may be useful for exploring diversity of Coniochaeta when the barcode locus (ITS rDNA) lacks sufficient resolution to differentiate species groups, and that these loci may be useful for inferring relationships within the genus more broadly (see also Friebes et al. 2016; Harrington et al. 2019). However, the present study highlights an ongoing challenge in ecological studies as well: when species proxies (operational taxonomic units) are based only on threshold levels of sequence similarity or divergence for barcode loci, cryptic species may be under-counted and estimates of diversity and host-/geographic-ranges will be limited.

The observation that conidial dimensions are highly plastic in this clade highlights the ongoing challenges with regard to taxonomy of Coniochaeta as a whole, in that morphological or whole-colony characters, particularly of anamorphs, can have limited value for resolving species. We found that three of the four species considered here produced fertile perithecia after extended periods of incubation that included three months of storage, suggesting that cold treatment may be useful in inducing ascomata formation in at least some lineages within Coniochaeta currently known only as anamorphs.

The lack of sexual structures in C. endophytica, first reported by Harrington et al. (2019) and again observed here, remains intriguing given the ready reproduction in vitro of the other species in the focal clade following similar treatment. Our phylogenetic analyses raise the possibility that $\mathrm{NC} 1642$ should be treated as a member of $C$. endophytica; Harrington et al. (2019) did not do so on the basis of distinctive morphology, phenotypes with respect to interactions with plant tissues, and most conclusively, differences at the genomic level (see Fig. 4 in Harrington et al. 2019). This isolate remains enigmatic, illustrating the biological and morphological diversity present among closely related species in this clade.

Overall, the present work illustrates the value of archiving fungi collected for ecological studies. Biodiversity collections that contain even unnamed or unidentified diversity represent important repositories. Integration of ecological and systematics efforts has the potential to re-shape our current ideas regarding the ecological distributions of diverse fungal species, their evolutionary relationships, and the structure and functional trait distributions across the highly diverse fungal tree of life. Here we illustrate this in one focal clade of Coniochaeta, a genus of great interest in studies of endophytes that occur in diverse temperate and boreal biomes in North America and beyond.

\section{Acknowledgments}

We thank the School of Plant Sciences, the Department of Ecology and Evolutionary Biology, and the College of Agriculture and Life Sciences at the University of Arizona for supporting this study. We thank the National Science Foundation for supporting the studies that led to the isolation of Coniochaeta endophytes from diverse hosts (to AEA, NSF DEB-1045766, DEB-1010675, DEB-1541496), and for supporting AHH via a Graduate Research Fellowship. We acknowledge NIFA for support to AEA and colleagues (ARZT-1361340-H25-242) and to the Robert L. Gilbertson Mycological Herbarium (ARZT1259370-S25-200). We thank Indigo Agriculture, Inc. for support relevant to the species description. We thank François Lutzoni and Jolanta Miadlikowska for co-leading field expeditions in which several of the focal strains were isolated, and we are grateful to local collaborators in each location. We are grateful to J. Carey, I. Kline, N. Yang, and C. Plecki for assistance in the laboratory, and especially to $\mathrm{M}$. Lee for project coordination and logistical support. The authors declare no conflict of interest in presenting this work.

\section{Author contributions}

Conceived of study: PI, AEA. Isolated focal strains, SO, JMU, AEA. Characterized morphology, AHH, AEA. Performed sequencing, AEA, SO, JMU. Conducted phylogenetic analyses: AEA. Wrote manuscript, AEA, with input from all authors. 


\section{Supplementary electronic material}

Figure S1. Single-locus tree, maximum likelihood analysis, ITSrDNA, including all of the 24 terminals in the final concatenated analysis. Download file

Figure S2. Single-locus tree, maximum likelihood analysis, $A C T$, including all of the 24 terminals in the final concatenated analysis. Download file

Figure S3. Single-locus tree, maximum likelihood analysis, GPD, including 22 of the 24 terminals in the final concatenated analysis. Download file

Figure S4. Single-locus tree, maximum likelihood analysis, $R P B 2$, including all of the ingroup taxa in the final concatenated analysis, but rooted internally because FL0068 and FL1226 were not amplified successfully. Download file

Figure S5. Single-locus tree, maximum likelihood analysis, TEF-1a, including 16 of the 24 terminals in the final concatenated analysis. Download file

Figure S6. Additional images of the holotype strains of C. lutea (A-C) and $C$. palaoa $(\mathrm{D}-\mathrm{F})$, grown as detailed in the main text. Scales: $\mathrm{A}=$ $100 \mu \mathrm{m} ; \mathrm{B}=50 \mu \mathrm{m} ; \mathrm{C}=25 \mu \mathrm{m} ; \mathrm{D}=200 \mu \mathrm{m} ; \mathrm{E}=40 \mu \mathrm{m} ; \mathrm{F}=30 \mu \mathrm{m}$. Download file

\section{References}

Agrawal, T. \& Kotasthane, A. S. 2012. Chitinolytic assay of indigenous Trichoderma isolates collected from different geographical locations of Chhattisgarh in central India. SpringerPlus 1: 73. https://doi. org/10.1186/2193-1801-1-73

Asgari, B., Zare, R. \& Gams, W. 2007. Coniochaeta ershadii, a new species from Iran, and a key to well-documented Coniochaeta species. Nova Hedwigia 84: 175-187. https://doi.org/10.1127/00295035/2007/0084-0175

Bills, G. F., González-Menéndez, V., Martín, J., Platas, G., Fournier, J., Peršoh, D. \& Stadler, M. 2012. Hypoxylon pulicicidum sp. nov. (Ascomycota, Xylariales), a pantropical insecticide-producing endophyte. PLoS One 7: e46687. https://doi.org/10.1371/journal.pone.0046687

Bussaban, B., Lumyong, S., Lumyong, P., Hyde, K. D. \& McKenzie, E. H. C. 2003. Three new species of Pyricularia are isolated as zingiberaceous endophytes from Thailand. Mycologia 95: 519-524. https://doi.org/10.2307/3761895

Carbone, I., White, J. B., Miadlikowska, J., Arnold, A. E., Miller, M. A., Kauff, F., U’Ren, J. M., May, G. \& Lutzoni, F. 2017. T-BAS: TreeBased Alignment Selector toolkit for phylogenetic-based placement, alignment downloads and metadata visualization: an example with the Pezizomycotina tree of life. Bioinformatics 33: 1160-1168. https://doi.org/10.1093/bioinformatics/btw808

Chen, K.-H., Miadlikowska, J., Molnár, K., Arnold, A. E., U’Ren, J. M., Gaya, E., Gueidan, C. \& Lutzoni, F. 2015. Phylogenetic analyses of eurotiomycetous endophytes reveal their close affinities to Chaetothyriales, Eurotiales and a new order - Phaeomoniellales. Molecular Phylogenetics and Evolution 85: 117-130. https://doi. org/10.1016/j.ympev.2015.01.008

Chen, K.-H. 2017. Evolution of fungal endophytes and their functional transitions between endophytism and saprotrophism. PhD Dissertation, Duke University, Durham, NC, USA.

Damm, U., Fourie, P. H. \& Crous, P. W. 2010. Coniochaeta (Lecythophora), Collophora gen. nov. and Phaemoniella species associated with wood necroses of Prunus trees. Persoonia - Molecular Phylogeny \& Evolution of Fungi 24: 60-80. https://doi. org/10.3767/003158510x500705

Del Olmo-Ruiz, M. 2012. Diversity, distributions, and host affiliations of fungal endophytes associated with seedless vascular plants ( $\mathrm{Ph} . \mathrm{D}$ dissertation). University of Arizona.

Edgar, R. C. 2004. MUSCLE: multiple sequence alignment with high accuracy and high throughput. Nucleic Acids Research 32: 1792-1797. https://doi.org/10.1093/nar/gkh340
Ewing, B., Hillier, L., Wendl, M. C. \& Green, P. 1998. Base-calling of automated sequencer traces with phred. Genome Research 8: 175-185. https://doi.org/10.1101/gr.8.3.175

Friebes, G., Jaklitsch, W. M., Garcia, S. \& Voglmayr, H. 2016. Lopadostoma taeniosporum revisted and a new species of Coniochaeta. Sydowia 68: 87-97.

Gams, W. \& McGinnis, M. R. 1983. Phialemonium, a new anamorph genus intermediate between Phialophora and Acremonium. Mycologia 75: 977-987. https://doi.org/10.1080/00275514.1983.12023783

García, D., Stchigel, A. M., Cano, J., Calduch, M., Hawksworth, D. L. \& Guarro, J. 2006. Molecular phylogeny of Coniochaetales. Mycological Research 110: 1271-1289. https://doi.org/10.1016/j.mycres.2006.07.007

Gazis, R., Rehner, S. \& Chaverri, P. 2011. Species delimitation in fungal endophyte diversity studies and its implications in ecological and biogeographic inferences. Molecular Ecology 20: 3001-3013. https://doi.org/10.1111/j.1365-294x.2011.05110.x

Gazis, R., Miadlikowska, J., Lutzoni, F., Arnold, A. E. \& Chaverri, P. 2012. Culture-based study of endophytes associated with rubber trees in Peru reveals a new class of Pezizomycotina: Xylonomycetes. Molecular Phylogenetics and Evolution 65: 294-304. https://doi. org/10.1016/j.ympev.2012.06.019

Han, J., Liu, C., L. Li, Zhou, H., Liu, L., Bao, L., Chen, Q., Song, F., Zhang, L., Li, E., Liu, L., et al. 2017. Decalin-containing tetramic acids and 4-Hydroxy-2-pyridones with antimicrobial and cytotoxic activity from the fungus Coniochaeta cephalothecoides collected in Tibetan Plateau (Medog). Journal of Organic Chemistry 82: 11474-11486. https://doi.org/10.1021/acs.joc.7b02010

Harrington, A. H., Del Olmo-Ruiz, M., U'Ren, J. M., Garcia, K., Pignatta, D., Wespe, N., Sandberg, D. C., Huang, Y.-L., Hoffman, M. T. \& Arnold, A. E. 2019. Coniochata endophytica sp. nov., a foliar endophyte associated with healthy photosynthetic tissue of Platycladus orientalis (Cupressaceae). Plant and Fungal Systematics 64: 65-79. https://doi.org/10.2478/pfs-2019-0008

Hoffman, M. T. \& Arnold, A. E. 2008. Geographic locality and host identity shape fungal endophyte communities in cupressaceous trees. Mycological Research 112: 331-344. https://doi.org/10.1016/j. mycres.2007.10.014

Huang, Y.-L., Devan, M. M. N., U’Ren, J. M., Furr, S. H. \& Arnold, A. E. 2016. Pervasive effects of wildfire on foliar endophyte communities in montane forest trees. Microbial Ecology 71: 452-468. https://doi.org/10.1007/s00248-015-0664-X

Huhndorf, S. M., Miller, A. N. \& Fernández, F. A. 2004. Molecular systematics of the Sordariales: the order and the family Lasiosphaeriaceae redefined. Mycologia 96: 368-387. https://doi.org/10. 1080/15572536.2005.11832982

Ivanová, H. \& Bernadovičová, S. 2012. New record of the fungus Coniochaeta prunicola on peaches from Slovakia. Biologia (Bratisl) 67: 269-273. https://doi.org/10.2478/s11756-012-0010-4

Kamiya, S., Uchiyama, S. \& Udagawa, S. 1995. Two new species of Coniochaeta with a cephalothecoid peridium wall. Mycoscience 36: 377-383. https://doi.org/10.1007/bf02268619

Khan, Z., Gene, J., Ahmad, S., Cano, J., Al-Sweih, N., Joseph, L., Chandy, R. \& Guarro, J. 2013. Coniochaeta polymorpha, a new species from endotracheal aspirate of a preterm neonate, and transfer of Lecythophora species to Coniochaeta. Antonie van Leeuwenhoek 104: 243-252. https://doi.org/10.1007/s10482-013-9943-z

Kirk, P., Cannon, P. F., Minter, D. W. \& Stalpers, J. A. 2008. Ainsworth \& Bisby's Dictionary of the Fungi. $10^{\text {th }}$ edn. CAB International, Wallingford, UK.

Maddison, W. P. \& Maddison, D. R. 2017. Mesquite: a modular system for evolutionary analysis. Version 3.2 .

Malloch, D. \& Cain, R. F. 1971. New cleistothecial Sordariaceae and a new family, Coniochaetaceae. Canadian Journal of Botany 49: 869-880. https://doi.org/10.1139/b71-127

Melin, E. \& Nannfeldt, J. A. 1934. Researches into the blueing of ground woodpulp. Svenska Skogsvårdsföreningens Tidskrift 32: 397-616. 
Nasr, S., Bien, S., Soudi, M. R., Alimadadi, N., Fazeli, S. A. S. \& Damm, U. 2018. Novel Collophorina and Coniochaeta species from Euphorbia polycaulis, an edemic plant in Iran. Mycological Progress 17: 755-771. https://doi.org/10.1007/s11557-018-1382-9

Oita, S., Carey, J., Kline, I., Ibáñez, A., Yang, N., Hom, E. F. Y., Carbone, I., U'Ren, J. M. \& Arnold, A. E. 2021. Methodological approaches frame insights into endophyte richness and community composition. Microbial Ecology 82: 21-34. https://doi.org/10.1007/ s00248-020-01654-y

Réblová, M., Miller, A. N., Rossman, A. Y., Seifert, K. A., Crous, P. W., Hawksworth, D. L., et al. 2016. Recommendations for competing sexual-asexually typified generic names in Sordariomycetes (except Diaporthales, Hypocreales, and Magnaporthales). IMA Fungus 7: 131-153. https://doi.org/10.5598/imafungus.2016.07.01.08

Rehner, S. \& Buckley, E. 2005. A Beauveria phylogeny inferred from nuclear ITS and EF1-a sequences: evidence for cryptic diversification and links to Cordyceps teleomorphs. Mycologia 97: 84-98. https://doi.org/10.1080/15572536.2006.11832842

Rojas, E. I., Herre, E. A., Mejía, L. C., Arnold, A. E., Chaverri, P. \& Samuels, G. J. 2008. Endomelanconiopsis, a new anamorph genus in the Botryosphaeriaceae. Mycologia 100: 760-775. https:// doi.org/10.3852/07-207

Rojas, E. I., Rehner, S. A., Samuels, G. J., Van Bael, S. A., Herre, E. A., Cannon, P., Chen, R., et al. 2010. Colletotrichum gloeosporioides s.l. associated with Theobroma cacao and other plants in Panama: multilocus phylogenies distinguish host-associated pathogens from asymptomatic endophytes. Mycologia 102: 1318-1338. https://doi. org/10.3852/09-244

Torres-Cruz, T. J., Billingsley, T. T. L., Almatruk, M., Hesse, C. N., Kuske, C. R., Desiro, A., et al. 2017. Bifiguratus adelaidae, gen. et sp. nov., a new member of Mucoromycotina in endophytic and soil-dwelling habitats. Mycologia 109: 363-378. https://doi.org/10. 1080/00275514.2017.1364958
U’Ren, J. M., Lutzoni, F., Miadlikowska, J., Laetsch, A. D. \& Arnold, A. E. 2012. Host and geographic structure of endophytic and endolichenic fungi at a continental scale. American Journal of Botany 99: 898-914. https://doi.org/10.3732/ajb.1100459

U'Ren, J. M., Lutzoni, F., Miadlikowska, J., Zimmerman, N. B., Carbone, I., May, G. \& Arnold, A. E. 2019. Host availability drives distributions of fungal endophytes in the imperiled boreal realm. Nature Ecology \& Evolution 3: 1430-1437. https://doi.org/10.1038/ s41559-019-0975-2

Vázquez-Campos, X., Kinsela, A. S., White, T. D., Collins, R. N. \& Neilan, B. A. 2014. Fodinomyces uranophilus gen. nov. sp. nov. and Coniochaeta fodinicola sp. nov., two uranium mine-inhabiting Ascomycota fungi from northern Australia. Mycologia 106: 1073-1089. https://doi.org/10.3852/14-013

Weber, E. 2002. The Lecythophora-Coniochaeta complex I. Morphological studies on Lecythophora species isolated from Picea abies. Nova Hedwigia 74: 159-185. https://doi.org/10.1127/00295035/2002/0074-0159

Weber, E., Görke, C. \& Begerow, D. 2002. The Lecythophora-Coniochaeta complex II . Molecular studies based on sequences of the large subunit of ribosomal DNA. Nova Hedwigia 74: 187-200.

Xie, J., Strobel, G. A., Feng, T., Ren, H., Mends, M. T., Zhou, Z. \& Geary, B. 2015. An endophytic Coniochaeta velutina producing broad spectrum antimycotics. Journal of Microbiology 53: 390-397. https://doi.org/10.1007/s12275-015-5105-5

Zwickl, D. J. 2006. Genetic algorithm approaches for the phylogenetic analysis of large biological sequence datasets under the Maximum Likelihood Criterion (PhD thesis). University of Texas at Austin. 